Published in final edited form as:

Arthritis Care Res (Hoboken). 2017 October ; 69(10): 1612-1616. doi:10.1002/acr.23173.

\title{
Prevalence of Primary Sjögren's Syndrome in a Population- Based Cohort in the United States
}

\author{
Gabriel Maciel, MD ${ }^{1,2}$, Cynthia S. Crowson, MS ${ }^{1,3}$, Eric L. Matteson, MD, MPH ${ }^{1,4}$, and Divi \\ Cornec, MD, PhD 5,6 \\ ${ }^{1}$ Division of Rheumatology, Mayo Clinic College of Medicine, Rochester, MN, USA \\ ${ }^{2}$ Hospital Maciel, 25 de Mayo 172, Montevideo, Montevideo, UY 11000 \\ ${ }^{3}$ Division of Biomedical Statistics and Informatics, Department of Health Sciences Research, \\ Mayo Clinic College of Medicine, Rochester, MN, USA \\ ${ }^{4}$ Division of Epidemiology, Department of Health Sciences Research, Mayo Clinic College of \\ Medicine, Rochester, MN, USA \\ ${ }^{5}$ Division of Pulmonary and Critical Care Medicine, Mayo Clinic College of Medicine, Rochester, \\ MN, USA \\ ${ }^{6}$ Rheumatology Department, Brest Teaching Hospital, Brest, France
}

\section{Abstract \\ Objective-To report the point prevalence of primary Sjögren's syndrome (pSS) in the first population-based study performed in the United States.}

\begin{abstract}
Methods-Cases of all potential pSS patients living in Olmsted County, Minnesota on January 1, 2015 were retrieved using the Rochester Epidemiology Project resources, and ascertained by manual medical record review. pSS cases were defined according to physician diagnosis. The use of diagnostic tests was assessed and the performance of classification criteria was evaluated. The number of prevalent cases in 2015 was also projected based on 1976-2005 incidence data from the same source population.
\end{abstract}

Results-A total of 106 patients with pSS were included in the study: 86\% were female, with a mean (SD) age of 64.6 (15.2) years and disease duration of 10.5 (8.4) years. A majority were antiSSA positive (75\%) and/or anti-SSB positive (58\%), but only 22\% met American-European Consensus Group or American College of Rheumatology criteria because the other tests required for disease classification were rarely performed in clinical practice (ocular dryness objective assessment, salivary gland functional or morphologic tests, or salivary gland biopsy). According to the physician diagnosis, age and sex adjusted prevalence of pSS was 10.3/10,000 inhabitants, but according to classification criteria this prevalence was only $2.2 / 10,000$. The analysis based on previous incidence data projected a similar 2015 prevalence rate of 11.0/10,000.

For Correspondence: Eric L. Matteson M.D., M.P.H., Divisions of Rheumatology and Epidemiology, Mayo Clinic College of Medicine, 200 1st St. S.W. Rochester, MN 55905 U.S.A. Matteson.eric@ mayo.edu. 
Conclusion-The prevalence of pSS in this geographically well-defined population was estimated between 2 and 10/10,000 inhabitants. Physicians rarely used tests included in the classification criteria to diagnose the disease in this community setting.

\section{Introduction}

Primary Sjögren's syndrome (pSS) is a systemic autoimmune disease characterized by an inflammatory infiltrate of exocrine (mostly lachrymal and salivary) glands. Cardinal symptoms include dryness of mouth and eyes, profound fatigue and wide-spread musculoskeletal pain, but up to one third of patients also experience extraglandular inflammatory involvement such as polysynovitis, neuropathy or inflammatory lung disease. No single test is diagnostic for the disease and several classification systems have been developed during the last 30 years for the study of pSS $(1,2)$.

Previous epidemiological studies have reported extraordinarily discrepant prevalence figures for pSS, ranging from $0.01 \%$ of the general population to more than $3 \%$, which has generated a debate as to whether pSS should be considered as a rare or frequent disease (3).

A recent meta-analysis of 18 prevalence studies computed an overall prevalence of $0.06 \%$ worldwide (4), but the heterogeneity of the studies included hindered the interpretation of these results. The main determinant factor explaining this variability was the methodology used in the different studies, with small sample questionnaire-based surveys (usually considered as low methodology quality) reporting high prevalence, and large populationbased studies reporting low prevalence estimates. No study previously reported the prevalence of pSS in the U.S. The only estimation of the prevalence of pSS in the U.S. was proposed by the National Arthritis Data Workgroup in 2005 (5), but was mostly extrapolated from prevalence studies performed in Greece and China.

The objective of this study was, therefore, to report the first prevalence estimates of pSS in the U.S., using a robust methodology in a carefully defined population.

\section{Methods}

\section{Case-finding methods}

Patients were selected from Olmsted County, a midwestern community in the state of Minnesota with 113,306 adult (age $\geq 18$ years) inhabitants as of $1 / 1 / 2015$. This population is well suited for investigation of the epidemiology of pSS. The Rochester Epidemiology Project (REP), a medical records linkage system, allows ready access to the complete (inpatient and outpatient) records from all healthcare providers for the local population, including the Mayo Clinic and its affiliated hospital, the Olmsted Medical Center and its affiliated community hospital, local nursing homes and a few private practitioners. The potential of this data system for population-based research and the generalizability of its results have been previously described (6). This system ensures virtually complete clinical information on all clinically recognized cases of pSS among Olmsted County residents.

The potential cases were all residents of Olmsted County, Minnesota on January first, 2015, and were selected based on diagnostic codes for Sjögren's syndrome, sicca syndrome and 
keratoconjunctivitis sicca (KCS). Fifty randomly selected patient records with diagnostic codes of xerostomia and 50 with KCS but without diagnosis of Sjögren's syndrome or relevant autoantibodies were also screened to assess whether any additional cases of pSS case were missed using the other codes. To exclude secondary Sjögren's syndrome patients, this first list was crossed electronically with datasets from existing REP cohorts of other systemic autoimmune diseases including rheumatoid arthritis, systemic lupus erythematosus, systemic sclerosis and inflammatory myopathies.

\section{Cases ascertainment}

Next, all individual clinical charts from patients selected during the first screening phase were reviewed. All patients were included who received a definite diagnosis of pSS in the opinion of the evaluating physicians, which were rheumatologists in almost all cases. The date of first pSS diagnosis was collected. Data were recorded regarding demographics, symptoms, and use and results of diagnostic tests such as Schirmer's test, ocular surface staining, salivary scintigraphy, parotid sialography, unstimulated salivary flow measurement, serological tests (antinuclear antibodies, anti-SSA and anti-SSB antibodies, rheumatoid factor, hypergammaglobulinemia) and minor salivary gland biopsy. Systemic involvement was analyzed according to the European League Against Rheumatism (EULAR) Sjögren's syndrome disease activity index (ESSDAI) score on the prevalence date, with no or low systemic activity category defined as an ESSDAI of less than 5, moderate activity as an ESSDAI comprised between 5 and 13, and high activity as an ESSDAI of 14 or more (7). Based on these data, patients who received a diagnosis of pSS in clinical practice were assessed to determine whether they fulfilled either of the two different classification criteria: the 2002 American-European Consensus Group (AECG) criteria (1) and the 2012 American College of Rheumatology (ACR) criteria (2).

\section{Statistical analyses}

Age- and sex-specific prevalence rates were calculated using the number of prevalence cases as the numerator and population estimates from the REP census as the denominator (8). Prevalence rates were age- and sex-adjusted to the 2010 U.S. white population. Ninety-five percent confidence intervals were computed for prevalence rates assuming that the observed number of cases follows a Poisson distribution.

The prevalence of pSS on January 1, 2015 was also estimated mathematically using the incident cohort of pSS cases diagnosed between 1976 and 2005 in Olmsted County (9). Annual incidence rates between 2006 and 2015 were projected using the overall annual incidence rate for the 1976-2005 time period. The prevalence of pSS was estimated by applying these age-, sex- and calendar year-specific disease incidence rates and the mortality rates from life tables to a hypothetical population, under the assumptions that the disease was not associated with any excess mortality as previously reported (9), and that migration in or out of the population was independent of disease status. Confidence intervals for the prevalence estimates were obtained using bootstrap methods. Analyses were performed using SAS version 9.4 (SAS Institute, Cary, NC, USA) and R 3.1.1 (R Foundation for Statistical Computing, Vienna, Austria). 


\section{Results}

\section{Characteristics of the population and performance of classification criteria in real-life patients}

A total of 106 patients with a physician-based diagnosis of pSS were living in Olmsted County on January 1, 2015 (Table 1). Most were women (86\%) and white (93\%), with a mean (SD) age of $64.6(15.2)$ years and a mean disease duration of 10.5 (8.4) years. The majority of patients (98\%) complained of dry mouth, dry eyes, or both. A vast majority of the patients $(85 \%)$ had no or low systemic activity at the time of the study according to the ESSDAI, $8 \%$ had moderate activity and $7 \%$ had high systemic activity. This systemic activity accounted mainly for the glandular, articular and biological domains.

Among the objective tests used by physicians to make a pSS diagnosis, serologic studies were the most frequently performed. Seventy percent of the patients with available results had positive anti-nuclear antibody, $75 \%$ had anti-SSA and 58\% anti-SSB antibodies, $49 \%$ had positive rheumatoid factor, and $49 \%$ had hypergammaglobulinemia. Conversely, objective explorations of sicca complaints were rarely performed. Ocular surface staining and Schirmer's test results were usually not specified in ophthalmologic visits reports, and were not used as a diagnostic tool by the rheumatologists. Salivary flow was almost never measured, and salivary scintigraphy or parotid sialography were performed in less than $10 \%$ of the patients. Minor salivary gland biopsy was performed in only 16 patients (positive in 10). Accordingly, only a minority (22\%) of the patients fulfilled AECG and ACR classification criteria. However, the main explanation for not fulfilling the criteria was that the required tests were not performed, and not that they were negative.

\section{Prevalence of pSS in the general population}

Overall, the age and sex adjusted prevalence of physician-diagnosed pSS was 10.3 per 10,000 inhabitants $(95 \%$ CI 8.3, 12.2, table 2). This figure was supported by further estimates based on 1976-2005 incidence data in Olmsted County, which projected a very similar 2015 prevalence of $11.0(95 \%$ CI 9.0,13.8) cases per 10,000 inhabitants. Prevalence of pSS by age and sex group confirmed the well described demographic characteristics of the disease, which affects primarily middle-age to older women. Using figures from the 2015 general U.S. population census, a total of 248,000 patients with pSS (35,000 males and 213,000 females) would currently live in the country. The prevalence of pSS cases which fulfilled the classification criteria was much lower at 2.2 cases per 10,000 inhabitants (95\% CI 1.3, 3.1), which would translate to an estimated total of 53,000 cases in the U.S.

\section{Discussion}

Using robust methods to enumerate the number of pSS cases in a well-defined population in the U.S., this study estimated the pSS prevalence between 2.2 and 10.3 per 10,000 inhabitants. This result has important consequences to better understand the epidemiology of the disease, as well as disease definition, classification, and diagnostic tools used by physicians in clinical practice. 
The results of previous population-based studies that reported pSS prevalence in other geographical areas are summarized in table 3 . The fact that the prevalence estimate from the current study is higher than those in other studies has several probable explanations. First, the broad screening methodology, using the REP, allowed complete full access to a performant coding system and to all individual medical records of the screened patients, permitting exhaustive case detection in the general population living in Olmsted County. Further, calculation of the 2015 prevalence estimate based on previous 1976-2005 incidence data from the same population obtained a result very similar to the actual "case-counting" method and strengthens the findings. Second, the definition of pSS cases used in this study, diagnosis made by the evaluating physician, even if not standardized, accurately represents the way the disease is currently diagnosed, and therefore treated, in actual practice in a community setting. Using only administrative coding systems without analyzing actual medical data from the clinical charts may lead to both inclusion of cases that do not have the diagnoses and failure to detect actual cases. On the other hand, using classification criteria to define cases may not be applicable in the community setting if tests included in the criteria are not used by the physicians. Finally estimates of pSS prevalence may be different in this population than in others because of geographical variations in disease prevalence due to different genetic backgrounds or environmental factors.

The results of this study highlight the conceptual differences between disease diagnosis and classification criteria. Classification criteria are tools designed specifically for clinical research. Their main objective is to guarantee that pSS populations included in different studies (especially clinical trials) will be similar and that a comparison of different studies will be possible. However, they are not designed to be used as clinical tools to diagnose the disease. Indeed, as the results of the current study clearly show, in a real-life community setting physicians rarely use several of the tests included in classification criteria for pSS to diagnose the disease in individual patients, drastically decreasing the sensitivity of classification criteria. It is possible that treating physicians in Olmsted County may overdiagnose the disease, but the general characteristics of this cohort are typical of a prevalent pSS cohort, notably the prevalence of anti-SSA/SSB antibodies.

In the absence of specific disease-modifying therapy for pSS, treatment is purely symptomatic in most patients. Therefore, patients with eye or mouth dryness (without systemic involvement) would receive similar care, whether they receive a diagnosis of pSS or not. In this context, physicians may consider that performing precise diagnostic tests would not be useful if their results would not change their prescription. However, many trials are currently ongoing in the disease, raising the hope of finally developing treatments with specific efficacy in pSS. Once such treatments are available, physicians may use more precise diagnostic procedures, to be able to recognize pSS with certainty among other causes of sicca symptoms. It may be anticipated that such a change in the approach to patients with suspected Sjögren's syndrome would affect epidemiological estimates of disease frequency and characteristics.

Based on these results, pSS may or may not formally be a "rare disease". Based upon the U.S. Rare Diseases Act of 2002, a rare disease is a condition that affects fewer than 200,000 people nationwide. We showed that the total number of patients living with pSS in the U.S. 
would be estimated between 53,000 (if we consider only cases fulfilling classification criteria) and 248,000 (considering all physician diagnosed cases). The European Commission on Public Health defines a rare disease as a condition with prevalence lower than 1 in 2,000 persons. Using figures from the U.S. population census of 2010 for inhabitants 18 years of age and older and the prevalence found in Olmsted County, the prevalence of pSS in the U.S. would be about 1:1,000 considering physician-diagnosed cases, but only about 1:5,000 if current classification criteria are used. This discussion of whether pSS is rare or not has important consequences for public health evaluation of the disease burden and for the development of future therapeutic agents for this indication. Future epidemiological studies will be required to definitely address these issues.

To conclude, this study is the first to report the prevalence of pSS in the US, which is estimated between 2.2 and 10.3 cases per 10,000 inhabitants in the general population of Olmsted County, which would represent 53,000 to 248,000 patients nationwide.

\section{Acknowledgments}

Funding: This work was partially funded by a grant from Roche Pharmaceuticals. This work was made possible using the resources of the Rochester Epidemiology Project, which is supported by the National Institute on Aging of the National Institutes of Health under Award Number R01AG034676 and CTSA Grant Number UL1 TR000135 from the National Center for Advancing Translational Sciences (NCATS), a component of the National Institutes of Health (NIH). The content is solely the responsibility of the authors and does not necessarily represent the official views of the National Institutes of Health.

\section{References}

1. Vitali C, Bombardieri S, Jonsson R, Moutsopoulos HM, Alexander EL, Carsons SE, et al. Classification criteria for Sjogren's syndrome: a revised version of the European criteria proposed by the American-European Consensus Group. Ann Rheum Dis. 2002; 61(6):554-8. [PubMed: 12006334]

2. Shiboski SC, Shiboski CH, Criswell L, Baer A, Challacombe S, Lanfranchi H, et al. American College of Rheumatology classification criteria for Sjogren's syndrome: a data-driven, expert consensus approach in the Sjogren's International Collaborative Clinical Alliance cohort. Arthritis Care Res (Hoboken). 2012; 64(4):475-87. [PubMed: 22563590]

3. Cornec D, Chiche L. Is primary Sjogren's syndrome an orphan disease? A critical appraisal of prevalence studies in Europe. Ann Rheum Dis. 2015; 74(3):e25. [PubMed: 25381229]

4. Qin B, Wang J, Yang Z, Yang M, Ma N, Huang F, et al. Epidemiology of primary Sjogren's syndrome: a systematic review and meta-analysis. Ann Rheum Dis. 2015; 74(11):1983-9. [PubMed: 24938285]

5. Helmick CG, Felson DT, Lawrence RC, Gabriel S, Hirsch R, Kwoh CK, et al. Estimates of the prevalence of arthritis and other rheumatic conditions in the United States. Part I. Arthritis Rheum. 2008; 58(1):15-25. [PubMed: 18163481]

6. St Sauver JL, Grossardt BR, Yawn BP, Melton LJ 3rd, Rocca WA. Use of a medical records linkage system to enumerate a dynamic population over time: the Rochester epidemiology project. Am J Epidemiol. 2011; 173(9):1059-68. [PubMed: 21430193]

7. Seror R, Bootsma H, Saraux A, Bowman SJ, Theander E, Brun JG, et al. Defining disease activity states and clinically meaningful improvement in primary Sjogren's syndrome with EULAR primary Sjogren's syndrome disease activity (ESSDAI) and patient-reported indexes (ESSPRI). Ann Rheum Dis. 2016; 75(2):382-9. [PubMed: 25480887]

8. St Sauver JL, Grossardt BR, Leibson CL, Yawn BP, Melton LJ 3rd, Rocca WA. Generalizability of epidemiological findings and public health decisions: an illustration from the Rochester Epidemiology Project. Mayo Clin Proc. 2012; 87(2):151-60. [PubMed: 22305027] 
9. Nannini C, Jebakumar AJ, Crowson CS, Ryu JH, Matteson EL. Primary Sjogren's syndrome 19762005 and associated interstitial lung disease: a population-based study of incidence and mortality. BMJ Open. 2013; 3(11):e003569.

10. Alamanos Y, Tsifetaki N, Voulgari PV, Venetsanopoulou AI, Siozos C, Drosos AA. Epidemiology of primary Sjogren's syndrome in north-west Greece, 1982-2003. Rheumatology (Oxford). 2006; 45(2):187-91. [PubMed: 16332955]

11. Eaton WW, Rose NR, Kalaydjian A, Pedersen MG, Mortensen PB. Epidemiology of autoimmune diseases in Denmark. J Autoimmun. 2007; 29(1):1-9. [PubMed: 17582741]

12. Goransson LG, Haldorsen K, Brun JG, Harboe E, Jonsson MV, Skarstein K, et al. The point prevalence of clinically relevant primary Sjogren's syndrome in two Norwegian counties. Scand J Rheumatol. 2011; 40(3):221-4. [PubMed: 21231797]

13. Sardu C, Cocco E, Mereu A, Massa R, Cuccu A, Marrosu MG, et al. Population based study of 12 autoimmune diseases in Sardinia, Italy: prevalence and comorbidity. PLoS One. 2012; 7(3):e32487. [PubMed: 22396771]

14. Yu KH, See LC, Kuo CF, Chou IJ, Chou MJ. Prevalence and incidence in patients with autoimmune rheumatic diseases: a nationwide population-based study in Taiwan. Arthritis Care Res (Hoboken). 2013; 65(2):244-50. [PubMed: 22899470]

15. Maldini C, Seror R, Fain O, Dhote R, Amoura Z, De Bandt M, et al. Epidemiology of primary Sjogren's syndrome in a French multiracial/multiethnic area. Arthritis Care Res (Hoboken). 2014; 66(3):454-63. [PubMed: 23983119] 


\section{Significance and Innovations}

1. This study reports the first prevalence rate of pSS in a well-defined population in the U.S.

2. Prevalence of physician-diagnosed pSS in Olmsted County, Minnesota was 10.3 cases per 10,000 inhabitants in 2015 .

3. This prevalence is higher than previous results obtained in other geographical areas, probably due to different methodological designs of the studies and definitions of the disease.

4. Current classification criteria do not reflect accurately the diagnosis of pSS in clinical practice, since they include tests that are rarely performed by the evaluating physicians. However, definition of the disease by physician diagnosis may overestimate its prevalence compared to more stringent definitions based on validated criteria. 


\section{Table 1}

Characteristics of 106 patients with prevalent primary Sjögren's syndrome living in Olmsted County on January 1, 2015

\begin{tabular}{|c|c|}
\hline Characteristic & Value* \\
\hline Age, years, mean (SD) & $64.6(15.2)$ \\
\hline Sex, Females & $91(86 \%)$ \\
\hline Ethnicity, White & $99(93 \%)$ \\
\hline Disease duration at prevalence, years, mean (SD) & $10.5(8.4)$ \\
\hline \multicolumn{2}{|l|}{ Smoking status at diagnosis of primary Sjögren's syndrome } \\
\hline Never & $59 / 103(57 \%)$ \\
\hline Current & $6 / 103(6 \%)$ \\
\hline Former & $38 / 103(37 \%)$ \\
\hline Ocular Symptoms & $94 / 103(91 \%)$ \\
\hline Oral symptoms & $85 / 96(89 \%)$ \\
\hline Rheumatoid factor test positive & $44 / 90(49 \%)$ \\
\hline Anti-Nuclear antibody test positive & $73 / 104(70 \%)$ \\
\hline Anti-SSA (anti-Ro) test positive & $73 / 97(75 \%)$ \\
\hline Anti-SSB (anti-La) test positive & $57 / 98(58 \%)$ \\
\hline Hypergammaglobulinemia present & $42 / 86(49 \%)$ \\
\hline Abnormal Ocular Staining (Van Bijsterveld score $\geq 4$ or Rose Bengal test) & $8 / 13(62 \%)$ \\
\hline Schirmer's test $\leq 5 / 5 \mathrm{~min}$ & $7 / 9(78 \%)$ \\
\hline Abnormal salivary scintigraphy or parotid sialography & $7 / 9(78 \%)$ \\
\hline Unstimulated salivary flow $\leq 0.1 \mathrm{ml} / \mathrm{min}$ & $1 / 1(100 \%)$ \\
\hline Histopathology positive & $10 / 16(63 \%)$ \\
\hline Met AECG criteria & $23(22 \%)$ \\
\hline Met ACR criteria & $23(22 \%)$ \\
\hline \multicolumn{2}{|l|}{ ESSDAI } \\
\hline Low disease activity $(<5)$ & $90(85 \%)$ \\
\hline Moderate disease activity (5-13) & $9(8 \%)$ \\
\hline High disease activity ( $\geq 14)$ & $7(7 \%)$ \\
\hline
\end{tabular}

Abbreviations: ESSDAI, European League Against Rheumatism (EULAR) Sjögren's syndrome disease activity index; AECG, 2002 AmericanEuropean Consensus Group; ACR, 2012 American College of Rheumatology 


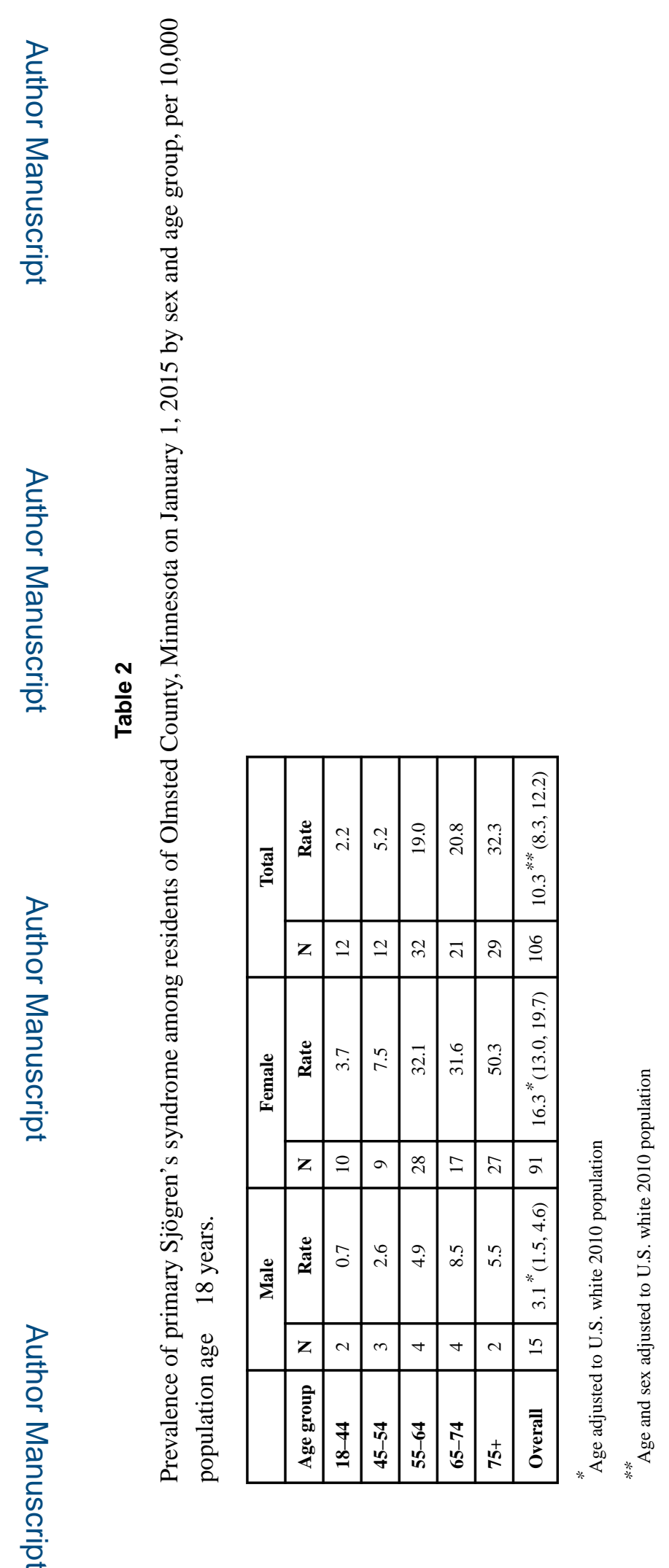

Arthritis Care Res (Hoboken). Author manuscript; available in PMC 2018 October 01. 


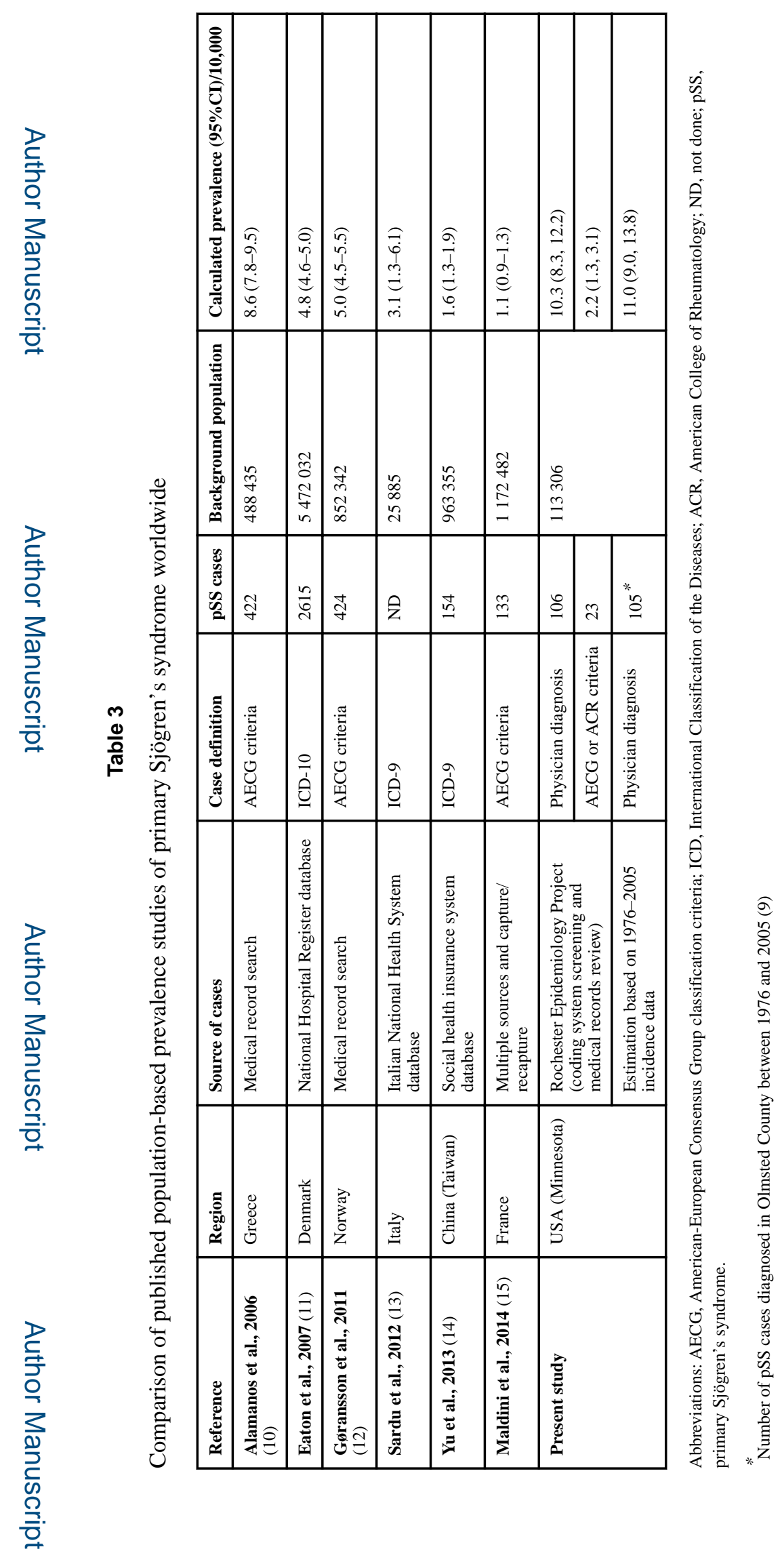

Arthritis Care Res (Hoboken). Author manuscript; available in PMC 2018 October 01. 